\title{
Gradhiva
}

Revue d'anthropologie et d'histoire des arts

11 | 2010

Grands hommes vus d'en bas

\section{Une vie de terrain au Mexique : les collections Stresser-Péan au musée du quai Branly}

A Lifetime's fieldworkin Mexico: the Stresser-Péan collections in the musée du quai Branly

Fabienne de Pierrebourg et Anne-Solène Rolland

\section{CpenEdition}

\section{Journals}

Édition électronique

URL : http://journals.openedition.org/gradhiva/1748

DOI : $10.4000 /$ gradhiva. 1748

ISSN : 1760-849X

\section{Éditeur}

Musée du quai Branly Jacques Chirac

Édition imprimée

Date de publication : 19 mai 2010

Pagination : 200-206

ISBN : 978-2-35744-025-8

ISSN : 0764-8928

\section{Référence électronique}

Fabienne de Pierrebourg et Anne-Solène Rolland, « Une vie de terrain au Mexique : les collections Stresser-Péan au musée du quai Branly », Gradhiva [En ligne], 11 | 2010, mis en ligne le 19 mai 2013, consulté le 21 avril 2019. URL : http://journals.openedition.org/gradhiva/1748; DOI : 10.4000/ gradhiva.1748 


\section{Une vie de terrain au Mexique : les collections Stresser-Péan au musée du quai Branly}

Fabienne de Pierrebourg Anne-Solène Rolland

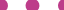

1. Dans le cadre de cette note, nous avons choisi de conserver l'ethnonyme Huastèque, bien qu'aujourd'hui leur propre dénomination, Teenek, soit plus couramment employée.

2. Elles sont conservées aujourd'hui au musée du quai Branly sous les numéros suivants : 71.1937.24 ; 71.1938.167 ; 71.1939.4.d; 71.1955.84.
Ethnologue, archéologue et ethnohistorien, Guy Stresser-Péan (1913-2009) a consacré sa vie au Mexique. Depuis 1936, année de sa première mission pour le musée de l'Homme, il a contribué, aidé de son épouse Claude qui l'a toujours accompagné dans ses recherches, qu'elle poursuit encore aujourd'hui, à enrichir considérablement la connaissance des rituels, des techniques et des objets des populations mexicaines. Il a laissé une collection de 3739 objets à présent conservés au musée du quai Branly. Issue de dons au musée de l'Homme puis au musée du quai Branly, celle-ci est le fruit de plus de soixante-dix années de terrain à travers le Mexique et le reflet d'intérêts constants pour la richesse des cultures matérielles rencontrées. Témoin rare des continuités et évolutions de ces dernières depuis la fin du xix et tout au long du xx siècle, elle est l'une des collections mexicaines les plus importantes d'Europe.

\section{L'époque du musée de l'Homme}

En 1936, Paul Rivet, qui domine le paysage de l'anthropologie française, remarque Stresser-Péan, alors étudiant, et lui propose d'aller au Mexique quand celuici était plus enclin à l'étude de la préhistoire africaine. Jacques Soustelle, qui avait également été envoyé au Mexique par Rivet, lui conseille de se consacrer à l'étude des Huastèques ${ }^{1}$ des régions chaudes de Tampico (Olivier 2008). Par la suite, il partage sa vie entre la France et le Mexique, où il finira ses jours dans sa maison de Mexico. Deux missions ethnographiques dans la région huastèque sont à l'origine des premières collections données au musée de l'Homme² : l'une se déroule entre 1936 et 1938; l'autre, retardée par la Seconde Guerre mondiale, entre 1950 et 1955. Stresser-Péan concentre d'abord ses recherches sur la culture 


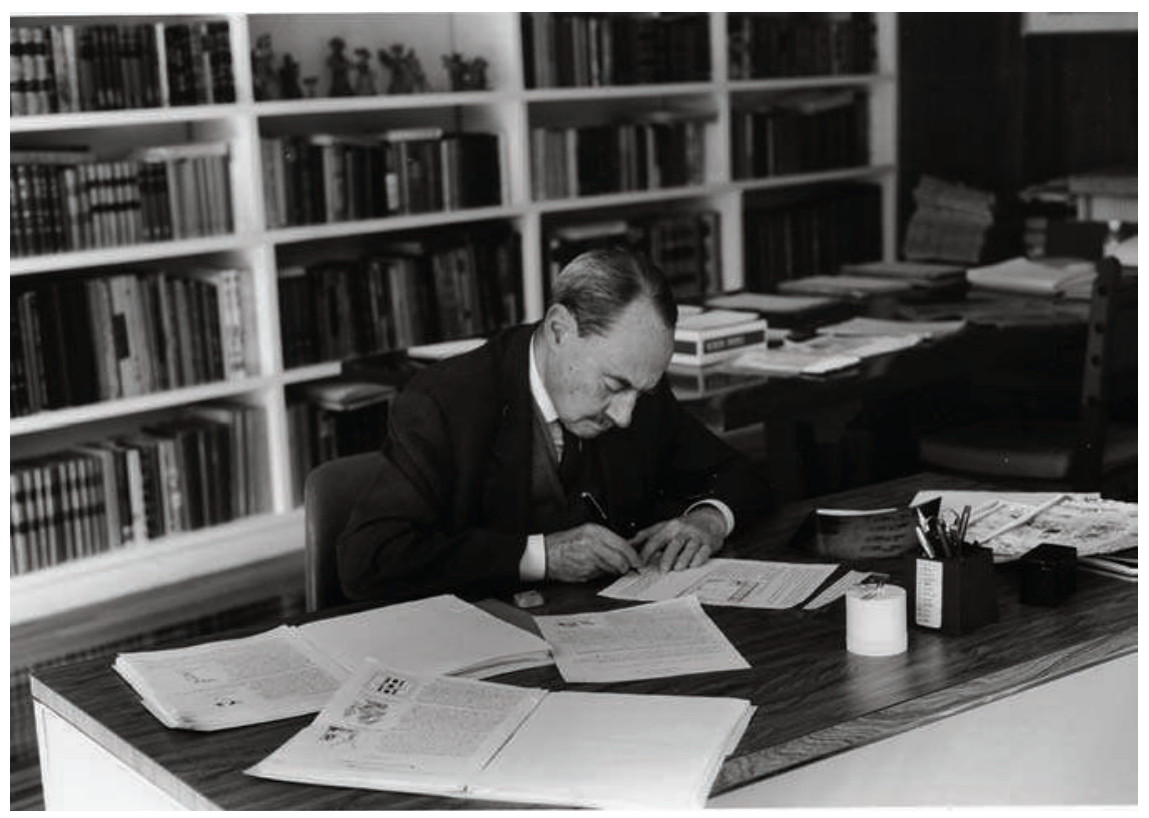

Fig. 1 Guy Stresser-Péan dans sa bibliothèque, Mexico, D.F., 1993. Photo Claude Stresser-Péan.

des Huastèques puis s'intéresse aux Nahuas habitant la même région, voyant entre les deux groupes une communauté de culture (Michelet 1989 : 20). Il étend ensuite ses études aux régions voisines et, au-delà, à toute la Mésoamérique.

"L'étude des Huastèques pouvait être abordée par l'archéologie, par l'ethnologie proprement dite, par la linguistique et par l'anthropologie.» (StresserPéan 1940 : 276) Stresser-Péan choisit l'ethnologie et la linguistique (enregistrements de textes et de vocabulaires) car, écrit-il encore : «L'archéologie a l'avenir devant elle, tandis que les faits ethnologiques et linguistiques qui subsistent encore de nos jours disparaissent rapidement dans le grand nivellement de la civilisation. " Il ne néglige pas pour autant les vestiges des cultures anciennes et réalise, parallèlement à ses enquêtes ethnographiques, des prospections accompagnées de relevés. Il rapporte un matériel considérable (plantes, insectes, photographies, films, plans, cartes, vocabulaires et objets) qu'il dépose dans différents laboratoires du Muséum national d'histoire naturelle.

À partir de 1956, sa carrière marque un tournant. Élu professeur à l'École pratique des hautes études (Sorbonne), il partage désormais sa vie entre l'enseignement en France et les missions au Mexique. En 1961, il crée la Mission archéologique et ethnologique française au Mexique (actuel Centre d'études mexicaines et centraméricaines), qui accueille à parts égales des travaux en archéologie et en ethnologie (Stresser-Péan 1967). Il en est le directeur jusqu'en 1977, s'installe de nouveau au Mexique et entame le second volet de sa carrière : il devient avant tout archéologue -il a mené son premier projet archéologique dans la région huastèque, à Tamtok, dès 1962. En 1965, il épouse Claude Stresser-Péan, qui l'accompagne dans tous ses travaux et travaille comme bénévole à la mission.

Ayant peu de temps à consacrer à l'ethnographie, qui demeure néanmoins l'un de ses principaux centres d'intérêt, Stresser-Péan délègue le travail de collecte à Alain Ichon, envoyé en région totonaque, et aux jeunes chercheurs accueillis à la mission, dont la spécialité et les directions de recherche se définissent dès cette époque. Ainsi, Jacques Galinier collecte des objets otomis, François Lartigues des 
objets tarahumaras, Alain Breton et Aurore Monod-Becquelin des objets tzeltals, Marie-Noëlle Chamoux et Danièle Dehouve des objets nahuas; lui-même et sa femme Claude collectent dans la région huastèque (Sevilla 2009). La mission s'était engagée à livrer à l'Institut national d'anthropologie et d'histoire du Mexique tout le produit de ses fouilles une fois l'étude terminée. Elle pouvait cependant envoyer librement en France les objets ethnographiques et les objets d'art populaire fabriqués par les Indiens dont elle étudiait les traditions. On opta pour cette dernière solution, et le fruit de ces collectes forme aujourd'hui la collection 71.1977.133, composée de 880 objets.

Les collections données au musée du quai Branly

En 1977, Stresser-Péan se retire de la direction de la mission, mais le couple continue ses recherches et son activité de collecte, et fait don dès 2000 au musée du quai Branly de plusieurs ensembles d'objets. Quatre d'entre eux sont des séries typologiques. La première série ${ }^{3}$, donnée au musée en 2001, est un ensemble de 1559 papiers découpés des populations otomis de l'État de Veracruz et des Nahuas de l'État de Puebla. Collectées entre les années 1930 et 1960, ces figures de papier industriel ou de papier d'écorce (amate) découpé étaient utilisées lors des rituels de divination et de fertilité agraire ou durant des séances thérapeutiques. La deuxième série 4 est composée d'un ensemble d'objets en bois laqué et des outils et matériaux nécessaires à leur fabrication provenant d'Olinala, dans l'État du Guerrero. Envoyé par Stresser-Péan dans ce village connu depuis le xviII siècle pour la décoration à l'huile de chia ${ }^{5}$ de boîtes et coffres, Arnaud Seydoux y réunit cette collection renseignant précisément sur la technique et l'iconographie de cette tradition, que les Stresser-Péan lui achètent dans les années 1960. La troisième collection ${ }^{6}$, plus anecdotique, est quant à elle constituée de 14 couples de puces habillées, achetées par Claude Stresser-Péan au musée des Arts et Industries populaires de Mexico en 1984. Expression originale propre aux paysans du Guanajuato installés à Mexico, la fabrication des puces ne perdura que quelques décennies au xxe siècle.

Enfin, l'ensemble le plus récent, donné par Guy et Claude Stresser-Péan au musée du quai Branly7, constitué de 555 objets, fait partie d'une importante collection encore en possession de Claude Stresser-Péan, qui travaille actuellement à une publication sur ces objets. Fruits de collectes effectuées depuis les années 1930 ou encore achetées à des particuliers, ces pièces proviennent pour l'essentiel de l'État d'Oaxaca et pour une moindre part du Chiapas. La collection comprend une majorité de textiles, zapotèques et tzotzils notamment, ainsi qu'une série de bijoux dont quelques pièces de l'ère coloniale, des objets du quotidien et des objets rituels. Par ces dons récents comme tout au long de leur carrière, Guy et Claude Stresser-Péant ont continué d'enrichir les collections mexicaines du musée du quai Branly.

\section{Les fils conducteurs de la collecte}

3. Collection 70.2001.40.

4. Collection 70.2004.3.

5. Salvia hispánica $L$.

6. Collection 70.2004.14.

7. Collection 70.2008.61.
Stresser-Péan fait partie des derniers chercheurs français à avoir étudié tant les cultures actuelles que préhispaniques. Ce double intérêt se reflète dans les objets recueillis, comprenant à la fois une collection archéologique issue des prospections menées durant les années 1930 et une collection ethnographique composée de plus de trois mille objets. Son intérêt pour les techniques remonte à ses années 


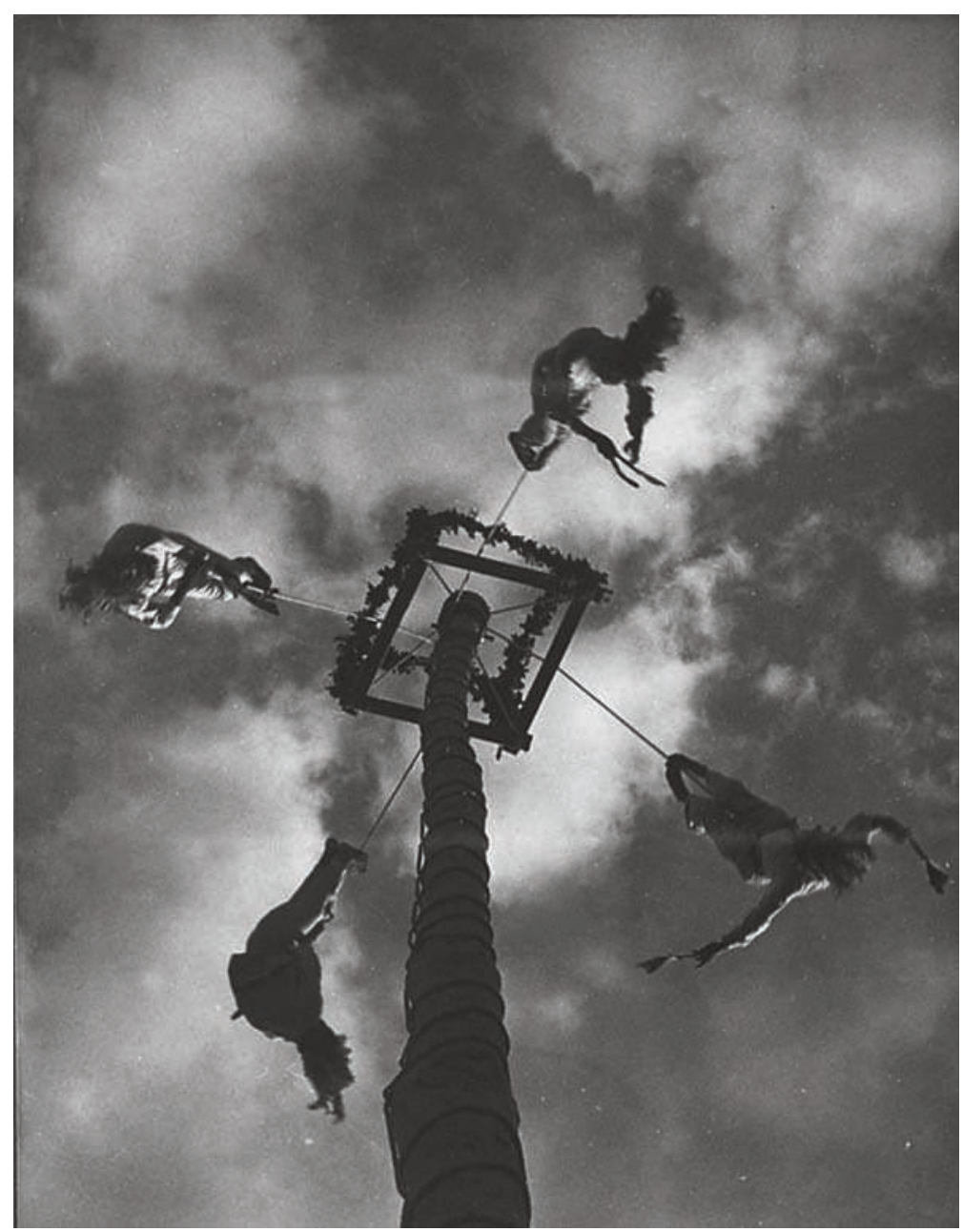

Fig. 2 Danse du Volador, descente des danseurs huastèques, Tamaletom, San Luis Potosí, 1951 @ musée du quai Branly, photo Guy Stresser-Péan.

d'étudiant et à l'influence du cours sur les «techniques du corps » de Marcel Mauss, puis à son premier contact avec l'histoire des techniques à travers la préhistoire, et a certainement été conforté par son amitié avec André-Georges Haudricourt (Olivier 2008 : 43). Les objets collectés - vanneries et textiles en cours de manufacture, objets de la vie quotidienne, témoins de techniques de pêche, de chasse, de portage, de consommation, etc. - reflètent cet intérêt particulier. Mais c'est surtout la collecte d'objets relatifs à la céramique qui en témoigne le mieux et lui permet, par ailleurs, de faire le lien entre le présent et l'histoire huastèque : échantillons d'argile et de dégraissant, outils de potier et objets finis collectés en série.

Durant ses premières missions, l'étude de la religion, des rituels et des danses est poussée aussi loin que possible grâce au recueil de nombreux mythes et pratiques rituelles. Une fois dégagé de ses obligations de directeur de la mission, Stresser-Péan continue à explorer ces sujets, principalement dans la région de la Sierra Norte de Puebla, et publie son dernier livre, Le Soleil-Dieu et le Christ (Stresser-Péan 2005b). Faute d'une liste exhaustive, nous ne citerons que quelques exemples parmi les très nombreux objets rituels présents dans les collections : cinq toiles d'araignée pour arrêter l'hémorragie, vaisselle miniature utilisée en médecine, idoles de papier, vêtements miniatures. Les instruments de 
musique, les costumes, les masques et les accessoires de danse sont probablement les objets les plus représentatifs de sa passion, en particulier ceux qui sont employés dans la danse du Volador, appelée localement «danse de l'aigle », qui, selon Stresser-Péan, serait une des rares danses actuelles ayant une origine préhispanique (ibid.).

Enfin, la collection que Stresser-Péan nous a transmise traduit une recherche constante des points de contact, des continuités, des ruptures et des transformations tant spatiales que chronologiques. S'il a choisi de poursuivre une longue recherche sur la région huastèque, c'est à la fois parce qu'elle représente, dit-il, l'extrémité septentrionale de la Mésoamérique mais aussi car «les Indiens huastèques présentent un intérêt particulier, parce qu'ils ont participé aux deux civilisations les plus élevées de l'Amérique indigène [maya et nahua] " (StresserPéan 1940 :276). Et refusant de se limiter à cette dernière région, il effectue ses derniers terrains dans la Sierra Norte de Puebla, qui a très probablement retenu son attention pour sa pluriethnicité : otomi, nahua et totonaque. Il a cherché à comprendre l'histoire d'une danse et d'une technique à travers leurs répétitions et leurs variantes dans d'autres cultures proches (nahua, otomi, tepehua) ou plus éloignées. Il a suivi la danse du Volador jusqu'au Guatemala et au Nicaragua. Ainsi, les collections ne sont pas limitées à la seule production des Indiens huastèques. De nombreuses autres cultures mésoaméricaines sont représentées, dont principalement les cultures nahua, zapotèque, mixtèque, otomi, mazatèque, tzeltal, tzotzil et lacandon.

\section{Les textiles dans les collections Stresser-Péan}

La très importante collection de textiles donnée au musée de l'Homme puis au musée du quai Branly contient à elle seule tous les aspects de la recherche du couple Stresser-Péan. Dès ses premières missions, en effet, Guy Stresser-Péan fait de la collecte et de la documentation des techniques textiles l'un des axes forts de son travail. En l'absence d'échantillons textiles préhispaniques dans la plupart des régions du Mexique, la collecte et l'étude des techniques indigènes, pour partie héritières de traditions disparues, lui semblent essentielles et urgentes, avant leur disparition. Dans son étude de la région huastèque, il consacre ainsi une part importante à la collecte et à la documentation du tissage du coton.

Cet axe se renforce lors de ses missions d'après-guerre, et notamment lors d'une enquête de terrain en 1952 dans la région de Huejutla, dans l'État d'Hidalgo, menée en compagnie d'Irmgard Weitlaner Johnson et de Bodil Christensen ${ }^{8}$, deux ethnologues spécialistes des textiles (Sevilla 2009). Première d'une longue

8. Ethnologue danoise spécialiste des textiles, Bodil Christensen est également à l'origine de la collection de textiles mexicains du musée national d'Ethnologie des Pays-Bas à Leyde.

9. Collection 71.1955.84.

10. 71.1955 .84 .552$.

11. Corsage.

12. 71. 1955.84 .615$.

13. 71.1961 .118$.

14. Collection 70. 2008.61. série de collaborations avec ces deux femmes, cette mission permet la collecte de nombreux textiles, donnés au musée de l'Homme en $1955^{\circ}$. Parmi les quelque trois cents textiles de cette collection, on compte plusieurs pièces remarquables, tels une jupe otomi à décor par teinture à réserve ${ }^{10}$ ou un huipil ${ }^{11}$ brodé de laine et de plumes ${ }^{12}$. Les textiles sont également un point fort d'une autre collection presque exclusivement textile ${ }^{13}$, constituée de cent pièces.

À partir des années 1980, la part des textiles dans les collections de Guy et Claude Stresser-Péan n'a cessé de croître. Le dernier don au musée du quai Branly ${ }^{14}$ est ainsi constitué très majoritairement de textiles, dont de nombreux costumes complets, issus de différentes périodes de collectes et d'achats. Grâce à l'ensemble de ces dons, le musée du quai Branly conserve aujourd'hui une collection impor- 


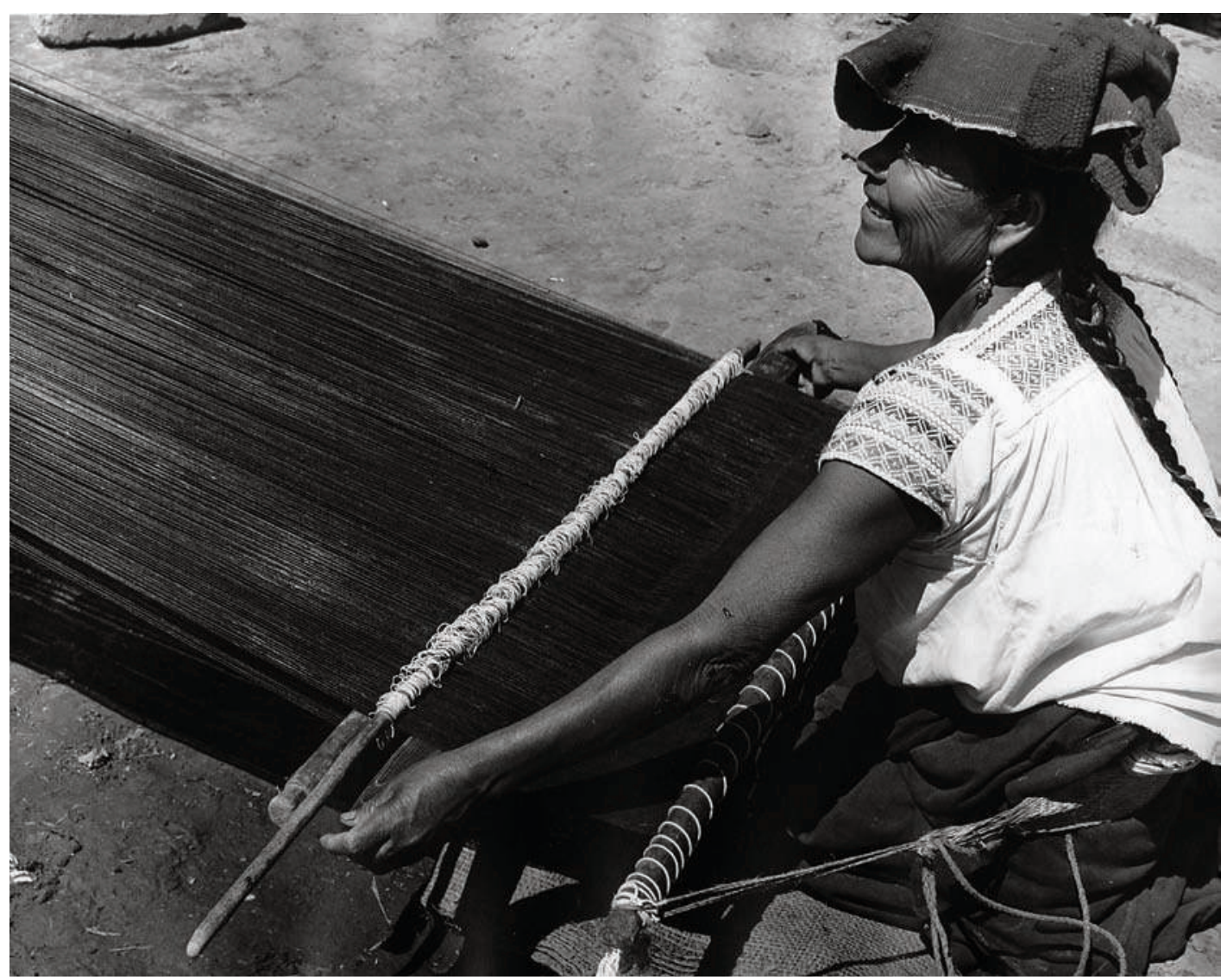

Fig. 3 Tisserande otomi, Santa Ana Hueytlalpan, Hidalgo, 1973. Photo Guy Stresser-Péan.

tante de textiles mexicains qui représente une très grande diversité de groupes ethniques, avec des points forts constitués notamment par les textiles de la région huastèque, de l'État d'Oaxaca et des Tzotzils du Chiapas. À cette diversité géographique correspond une grande variété de matériaux, de techniques et de formes vestimentaires : huipil, quechquemitl, rebozo, enredo... Par leur diversité et leur étendue chronologique, du xix siècle aux années 1990, ces collections constituent un précieux témoignage ethnologique et historique sur la richesse et les évolutions des vêtements traditionnels des populations indigènes du Mexique.

Fruits d'un très riche travail de recherches au Mexique, les collections StresserPéan sont donc le reflet à la fois de l'histoire de l'ethnologie française du xxe siècle et des évolutions techniques et culturelles des populations concernées. Par la diversité des sujets abordés - techniques, rituels, ethnomusicologie, textiles...-, elles offrent une matière inestimable à de futures recherches sur l'ethnologie du Mexique, qui viendront compléter le grand travail de collecte, de documentation et d'interprétation que Guy et Claude Stresser-Péan ont effectué.

musée du quai Branly / fdp@quaibranly.fr musée du quai Branly / anne.solene.rolland@gmail.com

mots clés / keywords : Mexique // Mexico • Stresser-Péan // Stresser-Péan • musée de l'Homme // musée de l'Homme - musée du quai Branly // musée du quai Branly•collections // collections • ethnologie // ethnology• archéologie // archeology $\cdot$ Huastèque // Huastec $\cdot$ textiles // textiles $\cdot$ rituels // rituals. 


\section{Ouvrages cités*}

\section{INSTRUCTIONS SOMMAIRES POUR LES COLLECTEURS D'OBJETS ETHNOGRAPHIQUES \\ 1931 Paris, musée d'Ethnographie du Trocadéro et mission Dakar-Djibouti, palais du Trocadéro.}

\section{León Portilla, Miguel}

2008 « Prólogo » in Guilhem Olivier (coord.), Viaje a la Huasteca con Stresser-Péan. Mexico, Centre d'études mexicaines et centraméricaines (Cemca), Fondo de Cultura Económica : 11-16.

\section{Michelet, Dominique}

1989 «En guise de présentation », in Dominique Michelet (coord.), Enquêtes sur l'Amérique moyenne. Mélanges offerts à Stresser-Péan. Mexico, INAH/CNCA/ CEMCA : 13-28.

\section{Olivier, Guilhem (coord.)}

2008 Viaje a la huasteca con Stresser-Péan.

Mexico, Centre d'études mexicaines et

centraméricaines, Fondo de Cultura Económica.

\section{Sevilia, Claudia de}

2009 Collections ethnographiques mexicaines du musée du quai Branly : une première approche historique, mémoire de recherche de $2^{\mathrm{e}}$ année de $2^{\mathrm{e}}$ cycle en histoire de l'art appliquée aux collections. Paris, École du Louvre (non publié).

\section{Stresser-PÉAn, Guy}

1940 «Mission de MM. Guy et Jacques StresserPéan au Mexique (1936-1938) », Journal de la Société des américanistes 32(2) : 275-284. 1947 La Danse du Volador chez les Indiens du Mexique et de l'Amérique centrale, mémoire. Paris, École pratique des hautes études (non publié).
1955 « Mission de MM. Guy et Jacques Stresser-Péan au Mexique (1950-1955) », Journal de la Société des américanistes 44[1] : 245-252.

1967 « Mission de MM. Guy et Jacques Stresser-Péan au Mexique (1962-1968) », Journal de la Société des américanistes 56[2] : 585-609.

$2005 a$ « El Volador. Datos históricos y simbolismo de la danza », Arqueologia Mexicana 13(75), septembre-octobre : 20-27. 2005b Le Soleil-Dieu et le Christ. La christianisation des Indiens du Mexique vue de la Sierra de Puebla. Paris, L'Harmattan.

\section{$\bullet \bullet$}

* Une bibliographie complète est consultable dans Michelet (1989).

\section{Résumé / Abstract}

Anne-Solène Rolland et Fabienne de Pierrebourg, Une vie de terrain au Mexique : les collections Stresser-Péan au musée du quai Branly - Ethnologue, archéologue et ethnohistorien, Guy Stresser-Péan (1913-2009) a consacré sa vie au Mexique. Grande figure de l'histoire de l'ethnologie française et de l'histoire commune de la France et du Mexique, il a laissé, aidé de son épouse Claude, une collection de 3739 objets aujourd'hui conservés au musée du quai Branly. Issues de dons au musée de l'Homme puis au musée du quai Branly, ces pièces sont le fruit de plus de soixante-dix ans de terrain à travers le Mexique et le reflet d'intérêts constants pour la richesse des cultures matérielles rencontrées. II ne s'agit pas ici de refaire une biographie de Stresser-Péan mais de suivre son itinéraire en tant que collecteur et de rendre hommage, quelques mois après son décès, à l'un des grands donateurs du musée du quai Branly, qui conserve l'une des collections mexicaines les plus importantes d'Europe.
Anne-Solène Rolland and Fabienne de Pierrebourg, A Lifetime's fieldwork in Mexico: the Stresser-Péan collections in the musée du quai Branly - Guy Stresser-Péan (1913-2009) was an ethnologist, archeologist and ethno-historian who devoted his life to Mexico. He was a major figure in the history of French ethnology, as well as France and Mexico's joint history, and he and his wife, Claude, left behind a collection of 3739 objects, today housed at the musée du quai Branly. These diverse objects, donated first to the musée de l'Homme and later to the musée du quai Branly, are the product of more than 70 years of fieldwork throughout Mexico and reflect Stresser-Péan's undiminished interest in the rich material cultures he encountered. This articleis not intended as a biographical contribution, but seeks to trace Guy Stresser-Péan's trajectory as a collector. It also pays hommage, a few short months after his death, to a man who was one of the musée du quai Branly's principal donator's and thanks to whom it houses one of the largest Mexican collections in europe. 